\title{
Heavy quark impact factor
}

\section{Michal Deak*}

Instituto de Física Corpuscular,

Universitat de València - Consejo Superior de Investigaciones Científicas,

Parc Científic, E-46980 Paterna (Valencia), Spain.

E-mail: michal.deak@ific.uv.es

\section{Grigorios Chachamis}

Instituto de Física Corpuscular,

Universitat de València - Consejo Superior de Investigaciones Científicas,

Parc Científic, E-46980 Paterna (Valencia), Spain.

E-mail: michal.deak@ific.uv.es

\section{German Rodrigo}

Instituto de Física Corpuscular,

Universitat de València - Consejo Superior de Investigaciones Científicas,

Parc Científic, E-46980 Paterna (Valencia), Spain.

E-mail: michal.deakeific.uv.es

We present the calculation of the finite part of the heavy quark impact factor at next-to-leading logarithmic accuracy in a form suitable for phenomenological studies at the LHC within the $k_{\perp}$ factorization scheme as described in the Ref. [1].

XXII. International Workshop on Deep-Inelastic Scattering and Related Subjects,

28 April - 2 May 2014

Warsaw, Poland

\footnotetext{
*Speaker.
} 


\section{Introduction}

One of the most important issues in QCD phenomenology at the Large Hadron Collider (LHC) is estimating the relevance of small- $x$ physics related effects on a number of physical observables and getting a definite answer on the validity and the applicability of the high energy resummation programme.

In the phase space region where the momentum exchanged is much smaller than the total center-of-mass energy, $|t| \ll s$ or equivalently at very small- $x, \log$ arithms in energy $(\log s \sim \log 1 / x)$ can spoil the convergence in the perturbative series of scattering amplitudes. More specificaly, terms of the form $\left(\alpha_{\mathrm{S}} \log 1 / x\right)^{n} \sim 1$, where $\alpha_{\mathrm{S}}$ is the strong coupling, for small enough $x$, need to be resummed to all orders.

The Balitsky-Fadin-Kuraev-Lipatov (BFKL) framework allows the resummation of centerof-mass energy logarithms at leading $[2,3,4](\mathrm{L} x)$ and next-to-leading $[5,6]$ accuracy (NL $x$ ). At $\mathrm{L} x$, all the terms of the form $\left(\alpha_{\mathrm{S}} \log 1 / x\right)^{n}$ need to be resummed whereas, at NLx one has also to resum terms in which the strong coupling lacks one power compared to the logarithm in energy, that is, terms that behave like $\alpha_{\mathrm{S}}\left(\alpha_{\mathrm{S}} \log 1 / x\right)^{n}$. The necessary ingredients for the study of scattering amplitudes within the BFKL formalism, are the gluon Green's function which is obtained by solving the BFKL equation, the gluon Regge trajectory and the impact factors, the latter being process-dependent objects. In a very general definition, the impact factors are the effective couplings of the scattering particles to whatever is exchanged in the $t$-channel for a process studied in the high energy factorization scheme. Resumming small- $x$ logarithms is finally well understood for a wide range of processes and observables at HERA and the LHC: perturbative evolution of parton distribution functions, photoproduction [7, 8, 9] and double-DIS [10, 11, 12] processes, Mueller-Navelet jets and forward jets in DIS.

The impact factors for gluons and massless quarks were calculated in Ref. [13] at NLx accuracy and in momentum space. This allows in principle for the calculation of various DIS and double-DIS processes with massless quarks and gluons in the initial state whereas the extension to the case of hadron-hadron collisions was also established.

\section{NL $x$ correction in an integral form}

The leading order quark impact factor is described by a simple formula

$$
h^{(0)}(k)=\sqrt{\frac{\pi}{N_{c}^{2}-1}} \frac{2 C_{F} \alpha_{S} N_{\varepsilon}}{k^{2} \mu^{2 \varepsilon}}, \quad N_{\varepsilon}=\frac{(4 \pi)^{\varepsilon / 2}}{\Gamma(1-\varepsilon)} .
$$

The NL $x$ impact factor can be written as a sum of the leading order impact factor and NL $x$ correction

$$
h_{\mathrm{q}}\left(k_{2}\right)=h^{(0)}\left(k_{2}\right)+h_{\mathrm{q}}^{(1)}\left(k_{2}\right)
$$

According to Ref. [1], the NL $x$ correction for the impact factor of a heavy quark can be written as the sum of three contributions:

$$
\begin{aligned}
h_{\mathrm{q}}^{(1)}\left(k_{2}\right) & =h_{\mathrm{q}, m=0}^{(1)}\left(k_{2}\right)+\int_{0}^{1} \mathrm{~d} z_{1} \int \mathrm{d}\left[k_{1}\right] \Delta F_{\mathrm{q}}\left(z_{1}, k_{1}, k_{2}\right) \\
& +\int \mathrm{d}\left[k_{1}\right] \bar{\alpha}_{\mathrm{S}} h_{\mathrm{q}}^{(0)}\left(k_{1}\right) K_{0}\left(k_{1}, k_{2}\right) \log \frac{m}{k_{1}} \Theta_{m k_{1}},
\end{aligned}
$$


with the convention $\Theta_{m k_{1}}=\theta\left(m-k_{1}\right)$, where the $\theta$-function is the well-known Heaviside step function and $k_{1}=\left|k_{1}\right|$. The first term on the right hand side of Eq.(2.3) is the NL $x$ correction to the impact factor of a massless quark, which can be expressed by using the leading order impact factor $h^{(0)}(k)$ and the gluon Regge trajectory $\omega^{(1)}(k)=-\frac{\bar{\alpha}_{\mathrm{S}}}{2 \varepsilon} \frac{\Gamma^{2}(1+\varepsilon)}{\Gamma(1+2 \varepsilon)}\left(\frac{k^{2}}{\mu^{2}}\right)^{\varepsilon}$,

$$
h_{\mathrm{q}, m=0}^{(1)}\left(k_{2}\right)=h^{(0)}\left(k_{2}\right) \omega^{(1)}\left(k_{2}\right)\left[b_{0}+\frac{3}{2}-\varepsilon\left(\frac{1}{2}+\mathscr{K}\right)\right] \text {, }
$$

with the beta function $b_{0}=\frac{11}{6}-\frac{n_{f}}{3 N_{c}}$ and $\mathscr{K}=\frac{67}{18}-\frac{\pi^{2}}{6}-\frac{5 n_{f}}{9 N_{c}}$.

The second term on the right hand side of Eq.(2.3) is the NL $x$ correction induced by the heavy quark mass $m$, with $\Delta F_{\mathrm{q}}\left(z_{1}, k_{1}, k_{2}\right)$ defined in Ref. [1]. The third term comes from the introduction of the mass scale to the leading order BFKL kernel $K_{0}\left(k_{1}, k_{2}\right)$ which is defined as

$$
\bar{\alpha}_{\mathrm{S}} K_{0}\left(k_{1}, k_{2}\right)=\frac{\bar{\alpha}_{\mathrm{S}}}{q^{2} \Gamma(1-\varepsilon) \mu^{2 \varepsilon}}+2 \omega^{(1)}\left(k_{1}\right) \delta[q], \quad \delta[q]=\pi^{1+\varepsilon} \delta^{2+2 \varepsilon}(q),
$$

with $q=k_{1}+k_{2}$. The first term on the right hand side of Eq.(2.4) corresponds the real component of the BFKL kernel and the second one to the virtual corrections. In the following text we shall analyze the second and third terms in the right hand side of Eq.(2.3).

\section{The $\Delta F_{\mathrm{q}}$ term}

The second term in the right hand side of Eq.(2.3),

$$
\Delta F_{\mathrm{q}}\left(k_{2}\right)=\int_{0}^{1} \mathrm{~d} z_{1} \int \mathrm{d}\left[k_{1}\right] \Delta F_{\mathrm{q}}\left(z_{1}, k_{1}, k_{2}\right)
$$

contains virtual and real corrections. The explicit expression of the integrand of Eq.(3.1) in momentum space (integrated over $k_{1}$ ) is given in Ref. [1]. The remaining integrations, however, cannot be performed directly in an straightforward way. It is easier to calculate the Mellin transform first, instead:

$$
\Delta \tilde{F}_{\mathrm{q}}(\gamma)=\Gamma(1+\varepsilon)\left(m^{2}\right)^{-\varepsilon} \int \mathrm{d}\left[k_{2}\right]\left(\frac{k_{2}^{2}}{m^{2}}\right)^{\gamma-1} \Delta F_{\mathrm{q}}\left(k_{2}\right),
$$

we get this expression

$$
\begin{aligned}
\Delta \tilde{F}_{\mathrm{q}}(\gamma) & =A_{\varepsilon}\left(m^{2}\right)^{\varepsilon} \frac{\Gamma(\gamma+\varepsilon) \Gamma(1-\gamma-2 \varepsilon) \Gamma^{2}(1-\gamma-\varepsilon)}{8 \Gamma(2-2 \gamma-2 \varepsilon)} \\
& \times\left[\frac{1+\varepsilon}{\gamma+2 \varepsilon}+\frac{2}{1-2 \gamma-4 \varepsilon}\left(\frac{1}{1-\gamma-2 \varepsilon}-\frac{1}{3-2 \gamma-2 \varepsilon}\right)\right] .
\end{aligned}
$$

Then, the function $\Delta F_{\mathrm{q}}\left(k_{2}\right)$ in momentum space is recovered by computing the inverse Mellin transform:

$$
\Delta F_{\mathrm{q}}\left(k_{2}\right)=\frac{1}{m^{2}} \int_{1-2 \varepsilon<\operatorname{Re} \gamma<1-\varepsilon} \frac{\mathrm{d} \gamma}{2 \pi i}\left(\frac{k_{2}^{2}}{m^{2}}\right)^{-\gamma-\varepsilon} \Delta \tilde{F}_{\mathrm{q}}(\gamma) .
$$

This integral is a contour integral in the complex plane which converges when the integration contour is parallel to the imaginary axis and intersects the real axis in the interval $1-2 \varepsilon<\operatorname{Re} \gamma<$ 
$1-\varepsilon$. To perform the integration in Eq.(3.4) we utilise the Cauchy residue theorem. The ratio $k_{2}^{2} / m^{2}$ takes, in principal, any value between 0 and $\infty$. However, if $k_{2}^{2} / m^{2}<1$, we deform the integration contour at $-i \infty$ and at $+i \infty$ to the right, such that the two ends meet at $+\infty$ of the real axis, whereas, if $k_{2}^{2} / m^{2}>1$ we deform the integration contour at $-i \infty$ and at $+i \infty$ to the left, in which case we denote the result by $\Delta F_{\mathrm{q}}^{-}\left(k_{2}\right)$.

In detail, after deforming the integration contour as described above, we have:

$$
\Delta F_{\mathrm{q}}^{-}\left(k_{2}\right)=\frac{1}{m^{2}} \sum_{\gamma \leq 1-2 \varepsilon} \operatorname{Res}\left[\left(\frac{k_{2}^{2}}{m^{2}}\right)^{-\gamma-\varepsilon} \Delta \tilde{F}_{\mathrm{q}}(\gamma)\right] \text {. }
$$

\section{The $K_{0}\left(k_{1}, k_{2}\right)$ related term}

Let us now turn to the final ingredient in order to have the full NL $x$ heavy quark impact factor with mass corrections. For the real emission part of the BFKL kernel, $K_{0}\left(k_{1}, k_{2}\right)$ (see Eq.(2.4)), we define the integral

$$
I_{m}\left(k_{2}\right)=\int \mathrm{d}\left[k_{1}\right] \frac{\bar{\alpha}_{\mathrm{S}} h_{\mathrm{q}}^{(0)}\left(k_{1}\right)}{q^{2} \Gamma(1-\varepsilon) \mu^{2 \varepsilon}} \log \frac{m}{k_{1}} \Theta_{m k_{1}} .
$$

We use the following integral representation [1]:

$$
\log \frac{a}{b} \Theta_{a b}=\lim _{\alpha \rightarrow 0^{+}} \int_{-i \infty}^{+i \infty} \frac{\mathrm{d} \lambda}{2 \pi i} \frac{1}{(\lambda+\alpha)^{2}}\left(\frac{a}{b}\right)^{\lambda} \equiv \int \mathrm{d}[\lambda]\left(\frac{a}{b}\right)^{\lambda},
$$

valid for $a, b>0$, which allows us to write

$$
I_{m}\left(k_{2}\right)=\frac{A_{\varepsilon}}{2} \lim _{\alpha \rightarrow 0^{+}} \int_{-i \infty}^{+i \infty} \frac{d \lambda}{2 \pi i} \frac{1}{(\lambda+\alpha)^{2}} \frac{\Gamma(1+\lambda-\varepsilon) \Gamma(\varepsilon) \Gamma(\varepsilon-\lambda)}{\Gamma(1+\lambda) \Gamma(2 \varepsilon-\lambda)}\left(m^{2}\right)^{\lambda}\left(k_{2}^{2}\right)^{-1-\lambda+\varepsilon} .
$$

The integrand in Eq.(4.3) vanishes for $|\lambda| \rightarrow \infty$ in all directions apart from the real axis. As was the case in the previous Section, this is a contour integral and can be calculated by a sum of residua in a way similar to 3.5 .

\section{The finite result}

The final NL $x$ impact factor can be written as

$$
h_{\mathrm{q}}\left(k_{2}\right)=\left.h_{\mathrm{q}}^{(1)}\left(k_{2}\right)\right|_{\text {sing }}+\left.h_{\mathrm{q}}\left(k_{2}\right)\right|_{\text {finite }},
$$

with the finite part of the result after the summation being:

$$
\begin{aligned}
\left.h_{\mathrm{q}}\left(k_{2}\right)\right|_{\text {finite }} & =h^{(0)}\left(k_{2}, \alpha_{\mathrm{S}}\left(k_{2}\right)\right)\left\{1+\frac{\alpha_{\mathrm{S}} N_{c}}{2 \pi}\left[\mathscr{K}-\frac{\pi^{2}}{6}+1-R \log (4 R)\right.\right. \\
& -\log (Z)\left((1+2 R) \sqrt{\frac{1+R}{R}}+2 \log (Z)\right) \\
& -3 \sqrt{R}\left(\mathrm{Li}_{2}(Z)-\mathrm{Li}_{2}(-Z)+\log (Z) \log \left(\frac{1-Z}{1+Z}\right)\right) \\
& \left.\left.+\mathrm{Li}_{2}(4 R) \Theta_{m k_{2}}+\left(\frac{1}{2} \log (4 R)+\frac{1}{2} \log ^{2}(4 R)+\mathrm{Li}_{2}\left(\frac{1}{4 R}\right)\right) \Theta_{k_{2} m}\right]\right\} .
\end{aligned}
$$

where $R=k_{2}^{2} /\left(4 m^{2}\right)$ and $Z=(\sqrt{1+R}+\sqrt{R})^{-1}$. 


\section{Numerical results and conclusions}

The result in Eq.(5.2) allows us to proceed to a first numerical study of the size of the mass corrections to the impact factor at NLx accuracy. We have adopted the running coupling scheme as described in Refs. $[14,15]$ with $n_{f}=5$ flavors. At L $x$ accuracy, we use a fixed value for the strong coupling constant $\bar{\alpha}_{\mathrm{S}}=0.2$.

In Fig. 1 we plot the Lx as well as the NLx quark impact factor, the latter for two quark mass values, $m=0$ and $m=5 \mathrm{GeV}$. We see that the NL $x$ correction to the leading order impact factor for massless quark is positive and moderate for small $k_{2}$ where the behavior is governed by the strong running coupling constant, but for most of the range of the plot the NL $x$ correction is negative. For a non-zero quark mass, in the region $k_{2}^{2} / \mathrm{m}^{2}<1$, the overall correction is positive and large. It turns negative closely after $k_{2}^{2} / m^{2}=1$, but for larger $k_{2}^{2} / m^{2}$, they follow the NL $x$ massless curve as expected. To get a better quantitative picture of the behavior of the NL $x$ corrections in the massless and massive case, it is useful to study the ratios of the impact factors at L $x$ and NL $x$ accuracy. In Fig. 2 (on the right) we can see that the relative size of the full NL $x$ corrections for small $k_{2}\left(k_{2}<10\right.$ $\mathrm{GeV}$ ) vary from more than $+100 \%$ down to some $-20 \%$, for similar mass values $m=4 \mathrm{GeV}$ and $m=5 \mathrm{GeV}$. To estimate the size of the NL $x$ corrections induced purely by the quark mass, we plot, Fig. 2 (on the left), the ratio between the finite parts of the NL $x$ massive and massless quark impact factor. The corrections are of the order of a $100 \%$ for the small $k_{2}^{2} / \mathrm{m}^{2}$ valeus and decrease as $k_{2}^{2} / \mathrm{m}^{2}$ is getting larger. The cusps in the curves are solely an artefact of the choice of the factorization scale. As expected, in the limit $k_{2} \rightarrow \infty$, the massless and massive NL $x$ impact factors coincide such that their ratio approaches 1 .

\section{Acknowledgments}

This work has been supported by the Research Executive Agency (REA) of the European Union under the Grant Agreement number PITN-GA-2010-264564 (LHCPhenoNet), by the Spanish Government and EU ERDF funds (grants FPA2011-23778 and CSD2007-00042 Consolider Project CPAN) and by GV (PROMETEUII/2013/007). GC acknowledges support from Marie Curie Actions (PIEF-GA-2011-298582). MD acknowledges support from Juan de la Cierva programme (JCI-2011-11382).

\section{References}

[1] G. Chachamis, M. Deak and G. Rodrigo, JHEP 1312, 066 (2013) [arXiv:1310.6611].

[2] L. N. Lipatov, Sov. J. Nucl. Phys. 23 (1976) 338;

[3] E. A. Kuraev, L. N. Lipatov, V. S. Fadin, Phys. Lett. B 60 (1975) 50, Sov. Phys. JETP 44 (1976) 443, Sov. Phys. JETP 45 (1977) 199.

[4] Ia. Ia. Balitsky, L. N. Lipatov, Sov. J. Nucl. Phys. 28 (1978) 822.

[5] V. S. Fadin, L. N. Lipatov, Phys. Lett. B 429 (1998) 127 [hep-ph/9802290];

[6] M. Ciafaloni, G. Camici, Phys. Lett. B 430 (1998) 349 [hep-ph/9803389].

[7] S. Catani, M. Ciafaloni and F. Hautmann, Phys. Lett. B 242, 97 (1990). 
[8] S. Catani, M. Ciafaloni and F. Hautmann, Nucl. Phys. B 366, 135 (1991).

[9] S. Catani and F. Hautmann, Nucl. Phys. B 427, 475 (1994) [hep-ph/9405388].

[10] S. J. Brodsky, F. Hautmann and D. E. Soper, Phys. Rev. Lett. 78, 803 (1997) [Erratum-ibid. 79, 3544 (1997)] [hep-ph/9610260].

[11] S. J. Brodsky, F. Hautmann and D. E. Soper, Phys. Rev. D 56, 6957 (1997) [hep-ph/9706427].

[12] J. Bartels, A. De Roeck and H. Lotter, Phys. Lett. B 389, 742 (1996) [hep-ph/9608401].

[13] M. Ciafaloni and D. Colferai, Nucl. Phys. B 538, 187 (1999) [hep-ph/9806350].

[14] G. Rodrigo and A. Santamaria, Phys. Lett. B 313 (1993) 441 [hep-ph/9305305];

[15] G. Rodrigo, A. Pich and A. Santamaria, Phys. Lett. B 424 (1998) 367 [hep-ph/9707474].

[16] S. Catani, B. R. Webber and G. Marchesini, Nucl. Phys. B 349, 635 (1991).

[17] Y. L. Dokshitzer, V. A. Khoze and S. I. Troian, Phys. Rev. D 53, 89 (1996) [hep-ph/9506425].

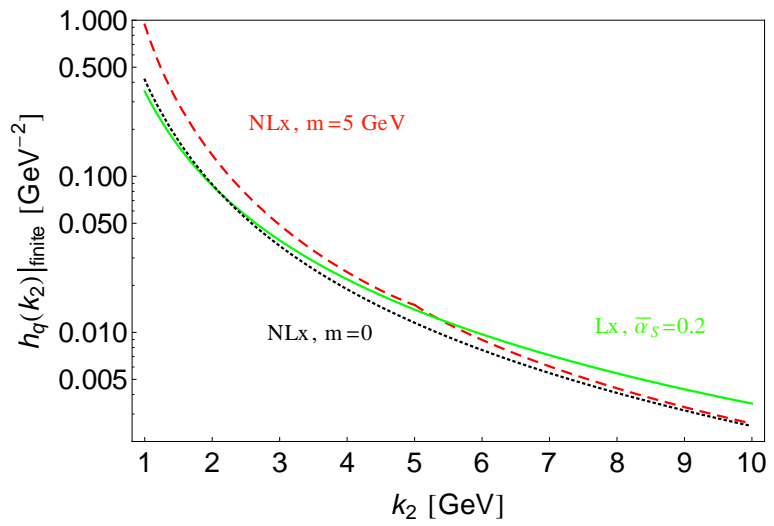

Figure 1: Transversal momentum distributions.
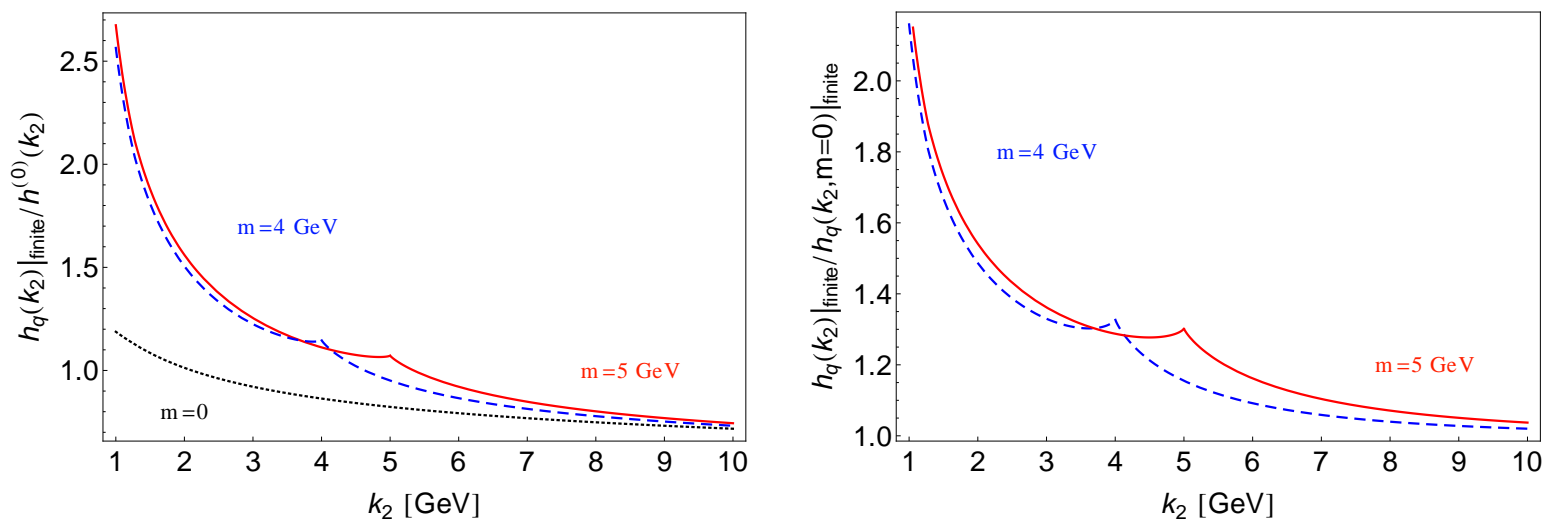

Figure 2: Transversal momentum dependence of ratios of impact factors. 